\title{
Improvement of Legionnaires' disease diagnosis using real-time PCR assay: a retrospective analysis, Italy, 2010 to 2015
}

Maria Luisa Ricci ${ }^{1}$, Antonella Grottola ${ }^{2,5}$, Giulia Fregni Serpini ${ }^{2}$, Antonino Bella ${ }^{1}$, Maria Cristina Rota ${ }^{1}$, Francesca Frascaro ${ }^{2}$,

Emanuela Pegoraro ${ }^{3}$, Marisa Meacci ${ }^{2}$, Anna Fabio ${ }^{2}$, Elena Vecchi ${ }^{4}$, Antonietta Girolamo ${ }^{1}$, Fabio Rumpianesi $^{2}$, Monica Pecorari², Maria Scaturro ${ }^{1}$

1. Department of Infectious Diseases, Istituto Superiore di Sanità, Rome, Italy

2. Unit of Microbiology and Virology, Polyclinic University Hospital, Modena, Italy

3. U.O.C. of Microbiology and Virology, Azienda Ospedaliero-Universitaria, Verona, Italy

4. Hospital Hygiene, Polyclinic University Hospital, Modena, Italy

5. Department of Surgery, Medicine, Dentistry and Morphological Scientists with Transplant Surgery, Oncology and Regenerative Medicine Relevance, University of Modena and Reggio Emilia, Modena, Italy

Correspondence: Maria Scaturro (maria.scaturro@iss.it)

Citation style for this article:

Ricci Maria Luisa, Grottola Antonella, Fregni Serpini Giulia, Bella Antonino, Rota Maria Cristina, Frascaro Francesca, Pegoraro Emanuela, Meacci Marisa, Fabio Anna, Vecchi Elena, Girolamo Antonietta, Rumpianesi Fabio, Pecorari Monica, Scaturro Maria. Improvement of Legionnaires' disease diagnosis using real-time PCR assay: a retrospective analysis, Italy, 2010 to 2015. Euro Surveill. 2018;23(50):pii=1800032. https://doi.org/10.2807/1560-7917.ES.2018.23.50.1800032

Aim: To evaluate real-time PCR as a diagnostic method for Legionnaires' disease (LD). Detection of Legionella DNA is among the laboratory criteria of a probable LD case, according to the European Centre for Disease Prevention and Control, although the utility and advantages, as compared to culture, are widely recognised. Methods: Two independent laboratories, one using an in-house and the other a commercial real-time PCR assay, analysed 354 respiratory samples from 311 patients hospitalised with pneumonia between 2010-15. The real-time PCR reliability was compared with that of culture and urinary antigen tests (UAT). Concordance, specificity, sensitivity and positive and negative predictive values (PPV and NPV, respectively) were calculated. Results: Overall PCR detected eight additional LD cases, six of which were due to Legionella pneumophila (Lp) non-serogroup 1. The two real-time PCR assays were concordant in $99.4 \%$ of the samples. Considering in-house real-time PCR as the reference method, specificity of culture and UAT was $100 \%$ and $97.9 \%$ (95\% Cl: 96.2-99.6), while the sensitivity was $63.6 \%(95 \% \mathrm{Cl}: 58.6-68.6)$ and $77.8 \%$ (95\% Cl: 72.9-82.7). PPV and NPV for culture were $100 \%$ and $93.7 \%$ (95\% Cl: 91.2-96.3). PPV and NPV for UAT were $87.5 \%$ (95\% Cl: 83.6-91.4) and 95.8\% (95\% Cl: 93.5-98.2). Conclusion: Regardless of the real-time PCR assay used, it was possible to diagnose LD cases with higher sensitivity than using culture or UAT. These data encourage the adoption of PCR as routine laboratory testing to diagnose LD and such methods should be eligible to define a confirmed LD case.

\section{Introduction}

Legionnaires' disease (LD) is a severe form of pneumonia and is caused by bacteria belonging to the Legionella genus. These microorganisms are ubiquitous in natural freshwater environments and can also thrive in man-made water systems. Legionella pneumophila (Lp) is the mostly responsible for the development of LD; serogroup 1 (sg1) is most frequently isolated from clinical samples [1]. LD cannot be clinically or radiologically distinguished from pneumonia cases of different aetiology, therefore the disease often remains undiagnosed. Age, underlying diseases, delay in diagnosis and inappropriate antibiotic therapy can result in an increase of the case fatality rate from LD [2].

In 2015 , the enumeration of all cases with a known outcome demonstrated an average case fatality rate of $8 \%$, with a higher rate $(28 \%)$ in nosocomial cases in Europe [3]. According to LD case definition [4,5], culture, a fourfold raise in Lp sg1 antibodies and urinary antigen test (UAT) are the only laboratory methods considered reliable for LD case confirmation. While serology has been nearly abandoned, UAT has almost completely replaced culture, representing $82 \%$ and 97\% of diagnosis in Europe and in the United States (US), respectively $[1,3]$. A similar trend was observed in Italy, where in 2016 UAT and culture were used to diagnose $95.5 \%$ and $2.7 \%$ of cases, respectively [6]. However, both culture and UAT have some limitations; culture is time consuming and has a sensitivity ranging from $<10-80 \%$ [1], UAT can be performed rapidly and with very high specificity for Lp sg1, but sensitivity for non-sg1 antigens is very low. In addition, the sensitivity 
Clinical samples analysed for admitted patients, Italy, 2010-15 ( $\mathrm{n}=311)$

\begin{tabular}{|l|c|c|c|c|}
\hline & $\begin{array}{c}\text { Number of tested } \\
\text { samples }\end{array}$ & $\begin{array}{c}\text { Number of positive } \\
\text { samples }\end{array}$ & $\begin{array}{c}\text { Number of negative } \\
\text { samples }\end{array}$ & $\begin{array}{c}\text { Number of individuals } \\
\text { tested }\end{array}$ \\
\hline In-house real-time PCR assay & 354 & 55 & 299 & 311 \\
\hline Commercial real-time PCR assay & 354 & 53 & 301 & 311 \\
\hline UAT & 278 & 40 & 238 & 246 \\
\hline Culture & 354 & 35 & 319 & 311 \\
\hline
\end{tabular}

PCR: polymerase chain reaction; UAT: urinary antigen test.

of UAT has been demonstrated to be lower for non-Lp sg1 MAb 3/1-positive strains [2]. Of note, laboratory diagnosis is often based on a single method, without taking into account the limitations that each diagnostic assay might have $[3,7]$.

Diagnostic tools based on detection of nucleic acids are an option to overcome the limitations observed by both culture and UAT. The numerous PCR assays proposed have shown high sensitivity and specificity, provided fast results and were able to detect a higher number of cases, giving the possibility to improve surveillance and better characterise local LD epidemiology [8-14]. Despite an increase in the proportion of cases diagnosed by PCR being reported in several European countries, the use of PCR is still very limited; presently a positive $P C R$ result only defines a LD probable case $[4,5]$. Currently, in Italy, only $0.1 \%$ of LD cases are diagnosed by PCR [6].

The aim of this retrospective study was to evaluate real-time $P C R$ as rapid diagnostic tool to define a LD case.

\section{Methods}

Respiratory samples were analysed using two different real-time PCR assays, performed in two different laboratories.

\section{Samples collection}

A total of 369 respiratory samples (including sputa, bronchial-alveolar lavages and bronchial aspirates) collected from 326 patients admitted to hospital for any pneumonia between 2010 and 2015 in Italy and were retrospectively analysed for Legionella pneumophila DNA detection.

Clinical samples were collected by two hospital laboratories, 74 samples (from 74 patients) from the Laboratory of Microbiology and Virology (University Hospital of Verona) and 295 (from 252 patients) from the Modena Regional Reference Laboratory (RRL) for Clinical Diagnosis of Legionellosis (Unit of Microbiology and Virology-Polyclinic University Hospital). All clinical samples were obtained 1 or 2 days after the onset of symptoms except three samples that were collected 5 days after onset of the disease. After collection, respiratory samples were stored at $-80{ }^{\circ} \mathrm{C}$ until tested.

Furthermore, 278 urine samples were available from 246 patients. There were 74 urine samples from 74 patients from Verona and 204 urine samples from 172 patients from Modena RRL.

Culture examination and urinary antigen test While patients were hospitalised with pneumonia symptoms, the Laboratory of Microbiology and Virology of Verona and the Modena RRL performed Legionella culture and UAT. For 25 patients culture was performed on two different respiratory samples and for nine patients on three samples, while for the remaining patients culture was performed on only one sample. Culture was carried out according to the procedures described elsewhere [15].

Both laboratories performed UAT by using both BinaxNOW Legionella Urinary Antigen Card kit and Binax Legionella Urinary Antigen EIA kit (Alere, Scarborough, US). Urine samples were always boiled before testing. For 19 patients UAT was performed on two urinary samples and for eight patients on three samples.

\section{Real-time PCR}

DNA extraction was performed at the Modena RRL using the ELITe STAR 200 Extraction kit (ELITechGroup S.p.A, Torino, Italy). DNA extracts were split in two aliquots to be analysed by real-time PCR at the Modena RRL and at the National Reference Laboratory at the Istituto Superiore di Sanità in Rome.

The Modena RRL analysed $5 \mu \mathrm{L}$ of DNA with the CE IVD marked real-time PCR commercial kit Legionella pn. Q-PCR Alert (ELITechGroup, CE IVD marked) detecting for Lp mip gene, according to the manufacturer's instructions on a 7300 Real-Time PCR System (Applied Biosystems, Foster City, California (CA), US). The NRL also analysed $5 \mu \mathrm{L}$ of DNA using an in-house real-time PCR assay in a final volume of $20 \mu \mathrm{L}$, containing $10 \mu \mathrm{L}$ of EXPRESS qPCR SuperMix, (Invitrogen, Carlsbad, CA, US), with Chromo 4 BioRad instrument (Bio-Rad, Hercules, CA, US), updated to CFX-96, and the following thermal protocol: 5 minutes at $95^{\circ} \mathrm{C}$ followed by 
TABLE 2

Legionnaires' disease cases with at least one positive diagnostic test, Italy, 2010-15 $(\mathrm{n}=52)$

\begin{tabular}{|l|c|c|c|} 
Culture & $\begin{array}{c}\text { Urinary } \\
\text { antigen test }\end{array}$ & $\begin{array}{c}\text { In-house real- } \\
\text { time PCR }\end{array}$ & $\begin{array}{c}\text { Number of cases } \\
\text { N }=52\end{array}$ \\
\hline Positive & Positive & Positive & 21 \\
\hline Positive & Negative & Positive & 3 \\
\hline Positive & ND & Positive & 5 \\
\hline Negative & Positive & Positive & 10 \\
\hline Negative & ND & Positive & 3 \\
\hline Negative & Negative & Positive & 5 \\
\hline Negative & Positive & Positive & 5 \\
\hline
\end{tabular}

ND: not done.

45 cycles of denaturation at $95^{\circ} \mathrm{C}$ for 10 seconds and annealing/extension at $60^{\circ} \mathrm{C}$ for 15 seconds. Primers and probes were as described by Mentasti et al. [14], targeting mip and wzm genes for detection of Lp (sg115) and sg1 marker, respectively. Primers and probes for internal control DNA were also as already described [14].

\section{Statistical analysis}

The concordance between tests was evaluated using the Kappa test ( $\mathrm{K}<0.20=$ "poor"; $0.20-0.40=$ "fair"; $0.40-0.60$ = "moderate"; $0.60-0.80$ = "good"; $0.80-$ $1.00=$ "very good"). The specificity, sensitivity and the positive and negative predictive values (PPV and NPV, respectively) and 95\% confidence intervals ( $\mathrm{Cl}$ ) for all methods were calculated considering the in-house real-time $P C R$ as a reference method. In addition, the concordance between all methods was also calculated. All statistical analyses were performed by Stata software version 11.2 (StataCorp, Texas, US).

\section{Results}

Samples analysed by real-time PCR, culture and urinary antigen test

Of 369 DNA samples, 15 were excluded from the comparison with culture and UAT because they were inhibitory in both PCR assays, as demonstrated by the absence of amplification of the internal control. These samples were also found negative for culture and UAT. Therefore, 354 samples from 311 patients were included in the comparison of PCR results with culture and/or UAT results (Table 1).

Both commercial and in-house real-time PCR assays gave the same results in 352 out of 354 samples, of which $299(85 \%)$ were negative and $53(15 \%)$ were positive (53 positive for mip marker and six positive also for wzm target). The in-house PCR detected two more positive samples $(n=55)$ compared with the commercial one. Of the 354 samples analysed by in-house PCR, six samples, (five negatives for both culture and UAT and one negative only for UAT but positive for culture) were identified as Lp non-sg1. Since the in-house PCR assay was able to differentiate Lp sg1 from the other serogroups, it was arbitrarily considered as a reference assay.

The concordance of the two PCR assays (commercial vs in-house) was $99.4 \%$ with a $K=0.98$ ( $p<0.0001$ ). Specificity and sensitivity of commercial PCR assay were calculated equal to $100 \%$ and $96.4 \%(95 \% \mathrm{Cl}$ : 94.4-98.3) respectively.

All 354 respiratory samples were also tested by culture; of these, 35 (9.9\%) were positive.

A total of 278 urine samples were tested by UAT and $40(14.3 \%)$ were found positive. The two methods used to detect the urinary antigen were concordant on all tested samples.

\section{Legionnaires' disease cases detected}

The total number of LD cases detected was 52 (Table 2) and it was calculated considering the patients with at least one positive diagnostic test (culture, UAT and $P(R)$. The in-house PCR assay was considered as a reference for comparison with culture and UAT results.

Using culture and/or urinary antigen test for diagnosis, the number of LD cases detected was 44; when the inhouse PCR assay was added, the number of detected cases increased to 52 (Table 2). PCR confirmed LD diagnosis in $84.6 \%$ of cases with at least one traditional diagnostic criterion positive (culture or UAT or both) and an increment of $18.2 \%$ was observed.

The comparison between culture and the in-house realtime PCR assay showed that the sensitivity of culture (63.6\%; 95\% Cl: 58.6-68.6) was lower, while the specificity was $100 \%$. The PPV and the NPV were $100 \%$ and 93.7\% (95\% Cl: 91.2-96.3), respectively. Overall concordance was good $(94.3 \% ; k=0.75 ; p<0.0001$ ) (Table 3).

The comparison between UAT and the in-house PCR showed a higher sensitivity ( $77.8 \% ; 95 \% \mathrm{Cl}: 72.9-82.7)$ than between culture and PCR, while specificity was slightly lower (97.9\%; 95\% Cl: 96.2-99.6), and PPV and NPV were $87.5 \%$ (95\% Cl: 83.6-91.4) and 95.8\% (95\% $\mathrm{Cl}$ : 93.5-98.2) respectively. Overall concordance of the two assays was good $(94.6 \% ; k=0.79 ; p<0.0001)$ (Table 3).

\section{Discussion}

In this study two independent laboratories, using a different real-time PCR assay for Legionella pneumophila DNA detection, analysed 354 respiratory samples and provided results with a very high concordance (99.4\%).

Our results highlight a higher sensitivity of PCR compared with culture and a higher diagnostic efficiency compared with UAT. Furthermore, as recently stressed 
Comparison of culture and UAT vs in-house real-time PCR by sensitivity, specificity, PPV, NPV, concordance and kappa value, Italy, 2010-15

\begin{tabular}{|c|c|c|c|c|c|c|c|}
\hline Comparison & Sensitivity $(95 \% \mathrm{Cl})$ & Specificity $(95 \% \mathrm{CI})$ & $\begin{array}{c}\text { PPV } \\
(95 \% \mathrm{Cl})\end{array}$ & $\begin{array}{c}\text { NPV } \\
(95 \% \mathrm{Cl})\end{array}$ & Concordance (\%) & $\begin{array}{l}\text { Kappa } \\
\text { value }\end{array}$ & $p$-value \\
\hline $\begin{array}{l}\text { Culture vs } \\
\text { real-time PCR in-house }\end{array}$ & $\begin{array}{c}63.6 \\
(58.6-68.6)\end{array}$ & 100.0 & 100.0 & $\begin{array}{r}93.7 \\
(91.2- \\
96.3) \\
\end{array}$ & $94 \cdot 3$ & 0.75 & $<0.0001$ \\
\hline $\begin{array}{l}\text { UAT vs } \\
\text { real-time PCR in-house }\end{array}$ & $\begin{array}{c}77.8 \\
(72.9-82.7)\end{array}$ & $\begin{array}{c}97.9 \\
(96.2-99.6)\end{array}$ & $\begin{array}{c}87.5 \\
(83.6-91.4)\end{array}$ & $\begin{array}{c}95.8 \\
\left(93.5^{-}\right. \\
98.2)\end{array}$ & 94.6 & 0.79 & $<0.0001$ \\
\hline
\end{tabular}

$\mathrm{Cl}$ : confidence interval; NPV: negative predictive value; PCR: polymerase chain reaction; PPV: positive predictive value; UAT: urinary antigen test.

by other authors $[16,17]$, it is important to perform more than one diagnostic assay in order to properly diagnose LD. Five of the eight LD cases with negative UAT results would have been missed if PCR assays, able to detect all Lp serogroups, had not been performed. Although in some instances UAT can incidentally detect non-1 Lp serogroups, they are designed to specifically detect Lp1 antigen, therefore, negative UAT results do not completely rule out LD infection. In addition to the aforementioned five cases (negative for UAT and for culture), three more culture-negative cases, resulted positive for Lp DNA by PCR. For these three, clinicians had only requested cultures and did not request UAT. Overall the eight additional cases show that even with a negative diagnosis but in presence of pneumonia, LD infection should be suspected and all available tests performed to investigate it.

Considering that urine samples were boiled before testing to destroy heat-sensitive proteins that could affect the test, false positive results can be reasonably excluded [7]. A possible explanation for the five UATpositive but PCR-negative cases was obtained querying patients' records: for two patients a sputum sample was promptly collected and analysed, while for the others sputum analysis was requested 5 or more days after the antibiotic therapy was started. Although there are not sufficient data to show if and how PCR results might be affected by an on-going antibiotic therapy, the above observation suggests the need to perform PCR assay as soon as possible, ideally before or immediately after the initiation of the antibiotic treatment.

The NPV was suggestive of the excellent reliability of the PCR methods, even though only Lp DNA was targeted. However, this limitation can often be found also using culture method, because specific and selective Legionellaisolation media, such as buffered charcoal yeast extract (BCYE) and glycine vancomycin polymyxin cycloheximide (GVPC), poorly support Legionella non-pneumophila growth [18]. The
PPV was also consistent with a higher sensitivity of PCR than culture.

The reliability of PCR in diagnosing LD is more and more recognised by the scientific community and recent studies demonstrated a better performance of PCR compared with other diagnostic assays, regardless of the type of respiratory sample (bronchoalveolar lavage or sputum) $[9,13]$. Moreover, PCR can also detect the presence of all Legionella species some of which are notoriously difficult to isolate by culture [19].

In this study, the use of real-time PCR resulted in an increment of eight (18.2\%) identified LD cases and therefore is an objective improvement in the diagnosis of LD. Real-time PCR has been considered a poorly reliable method due to the risk of cross-contaminations, however, the introduction of automated procedures for DNA extractions and also for PCR set up, has resulted in a consistent improvement in preventing this PCR drawback. Therefore, after an appropriate validation of their own molecular tests, clinical microbiology laboratories can adopt PCR assays to detect Legionella in respiratory samples.

The adoption of rapid methods to quickly identify LD cases is a priority, as the infection rate is underestimated all over the world and difficult to quantify, and increasing in several countries $[2,3,20]$. The laboratory procedures currently used to define confirmed LD cases are not able to guarantee a high level of sensitivity and specificity of results and they can be time-consuming. As a rapid LD diagnosis is crucial for both patient management and public health purposes, real-time PCR should be considered and implemented both locally and at Legionella reference laboratories in combination with all the other available methods.

In conclusion, as already observed in other countries, this study shows that the introduction of real-time PCR can improve LD diagnosis and should be considered among the criteria to define confirmed cases of LD [13]. 


\section{Acknowledgements}

We are grateful to Massimo Mentasti for his precious comments and editing of the manuscript.

This work was supported by the Ministry of Health (Centro per il controllo delle malattie, 2013-2014; Grant N. 5M12).

\section{Conflict of interest}

None declared.

\section{Authors' contributions}

Maria Scaturro, Maria Luisa Ricci, Monica Pecorari and Antonella Grottola designed the study. Antonella Grottola, Giulia Fregni Serpini, Francesca Frascaro, Antonietta Girolamo and Maria Scaturro performed the real-time PCR experiments. Antonella Grottola, Giulia Fregni Serpini, Francesca Frascaro, Monica Pecorari, Maria Luisa Ricci and Maria Scaturro elaborated the results. Emanuela Pegoraro provided respiratory samples and analysed them by culture; Emanuela Pegoraro also provided urinary antigen test data. Marisa Meacci and Anna Fabio analysed respiratory samples by culture and urine by urinary antigen test. Elena Vecchi and Monica Pecorari queried patients' records. Antonino Bella and Maria Cristina Rota performed the statistical analysis. Maria Scaturro wrote the manuscript and Antonella Grottola and Giulia Fregni Serpini helped her with the editing. All authors read and approved the final version of the manuscript.

\section{References}

1. Mercante JW, Winchell JM. Current and emerging Legionella diagnostics for laboratory and outbreak investigations. Clin Microbiol Rev. 2015;28(1):95-133. https://doi.org/10.1128/ CMR.00029-14 PMID: 25567224

2. Phin N, Parry-Ford F, Harrison T, Stagg HR, Zhang N, Kumar K, et al. Epidemiology and clinical management of Legionnaires disease. Lancet Infect Dis. 2014;14(10):1011-21. https://doi. org/10.1016/S1473-3099(14)70713-3 PMID: 24970283

3. European Centre for Disease Prevention and Control (ECDC). Annual Epidemiological report for 2015. Legionnaires' disease. Stockholm: ECDC; 2017. Available from: https://ecdc.europa.eu/en/publications-data/ legionnaires-disease-annual-epidemiological-report-2015

4. European Centre for Disease Prevention and Control (ECDC). European Union (EU) case definitions. Stockholm: ECDC; 2018. Available from: https://ecdc.europa.eu/en/infectious diseases-public-health/surveillance-and-disease-data/ eu-case-definitions

5. Centers for Disease Control and Prevention (CDC). 2012 National Notifiable Infectious Diseases (Historical). Atlanta: CDC; 2012 . Available from: https://wwwn.cdc.gov/nndss/ conditions/notifiable/2012/infectious-diseases/

6. L'Istituto Superiore di Sanità (ISS). La legionellosi in Italia nel 2017. [Legionellosis in Italy in 2017]. Rome: ISS; 2018. Italian. Available from: http://old.iss.it/binary/publ/cont/ONLINE.pdf

7. Rota MC, Fontana S, Montaño-Remacha C, Scaturro M, Caporali MG, Vullo V, et al. Legionnaires' disease pseudoepidemic due to falsely positive urine antigen test results. J Clin Microbiol. 2014;52(6):2279-80. https://doi.org/10.1128/JCM.00493-14 PMID: 24719437

8. Avni T, Bieber A, Green H, Steinmetz T, Leibovici L, Paul M. Diagnostic Accuracy of PCR Alone and Compared to Urinary Antigen Testing for Detection of Legionella spp.: a Systematic Review. J Clin Microbiol. 2016;54(2):401-11. https://doi. org/10.1128/JCM.02675-15 PMID: 26659202

9. Botelho-Nevers E, Grattard F, Viallon A, Allegra S, Jarraud S, Verhoeven $\mathrm{P}$, et al. Prospective evaluation of RT-PCR on sputum versus culture, urinary antigens and serology for Legionnaire's disease diagnosis. J Infect. 2016;73(2):123-8. https://doi. org/10.1016/j.jinf.2016.04.039 PMID: 27306488

10. Chen DJ, Procop GW, Vogel S, Yen-Lieberman B, Richter SS. Utility of PCR, Culture, and Antigen Detection Methods for Diagnosis of Legionellosis. J Clin Microbiol. 2015;53(11):3474-7. https://doi.org/10.1128/JCM.01808-15 PMID: 26292304
11. Benitez AJ, Winchell JM. Clinical application of a multiplex real-time PCR assay for simultaneous detection of Legionella species, Legionella pneumophila, and Legionella pneumophila serogroup 1. J Clin Microbiol. 2013;51(1):348-51. https://doi. org/10.1128/JCM.02510-12 PMID: 23135949

12. Murdoch DR. Diagnosis of Legionella infection. Clin Infect Dis. 2003;36(1):64-9. https://doi.org/10.1086/345529 PMID: 12491204

13. Murdoch DR, Podmore RG, Anderson TP, Barratt K, Maze MJ, French KE, et al. Impact of routine systematic polymerase chain reaction testing on case finding for Legionnaires' disease: a pre-post comparison study. Clin Infect Dis. 2013;57(9):1275-81. https://doi.org/10.1093/cid/cit504 PMID: 23899682

14. Mentasti M, Kese D, Echahidi F, Uldum SA, Afshar B, David $S$, et al. Design and validation of a qPCR assay for accurate detection and initial serogrouping of Legionella pneumophila in clinical specimens by the ESCMID Study Group for Legionella Infections (ESGLI). Eur J Clin Microbiol Infect Dis. 2015;34(7):1387-93. https://doi.org/10.1007/s10096-015-23634 PMID: 25851812

15. Istituto Superiore di Sanità (ISS). Linee guida per la prevenzione ed il controllo della legionellosi [Guidelines for prevention and control of legionellosis]. Rome: ISS; 2015. Italian. Available from: http://old.iss.it/binary/iss4/cont/C_17_ pubblicazioni_2362.pdf

16. Peci A, Winter AL, Gubbay JB. Evaluation and Comparison of Multiple Test Methods, Including Real-time PCR, for Legionella Detection in Clinical Specimens. Front Public Health. 2016;4:175. https://doi.org/10.3389/fpubh.2016.00175 PMID: 27630979

17. Svarrer CW, Lück C, Elverdal PL, Uldum SA. Immunochromatic kits Xpect Legionella and BinaxNOW Legionella for detection of Legionella pneumophila urinary antigen have low sensitivities for the diagnosis of Legionnaires' disease. J Med Microbiol. 2012;61(2):213-7. https://doi.org/10.1099/jmm.0.035014-0 PMID: 21921112

18. Lee JV, Lai S, Exner M, Lenz J, Gaia V, Casati S, et al. An international trial of quantitative PCR for monitoring Legionella in artificial water systems. J Appl Microbiol. 2011;110(4):103244. https://doi.org/10.1111/j.1365-2672.2011.04957.x PMID: 21276147

19. Vaccaro L, Izquierdo F, Magnet A, Hurtado C, Salinas MB, Gomes TS, et al. Correction: First Case of Legionnaire's Disease Caused by Legionella anisa in Spain and the Limitations on the Diagnosis of Legionella non-pneumophila Infections. PLoS One. 2016;11(9):e0162934. https://doi.org/10.1371/journal. pone.0162934 PMID: 27607064

20. Farnham A, Alleyne L, Cimini D, Balter S. Legionnaires' disease incidence and risk factors, New York, New York, USA, 20022011. Emerg Infect Dis. 2014;20(11):1795-802. https://doi. org/10.3201/eid2011.131872 PMID: 25513657

\section{License and copyright}

This is an open-access article distributed under the terms of the Creative Commons Attribution (CC BY 4.0) Licence. You may share and adapt the material, but must give appropriate credit to the source, provide a link to the licence, and indicate if changes were made.

This article is copyright of the authors or their affiliated institutions, 2018. 